\section{Systemische Mastozytose - Klassifikation, Symptome, assoziierte Erkrankungen, aktuelle Therapietrends}

\author{
Zwei Falldarstellungen
}

\author{
C. Rogalski ${ }^{1}$, U.-F. Haustein ${ }^{1}$, U. Paasch ${ }^{1}$, \\ B. Schmoranzer ${ }^{1}$, T. Friedrich ${ }^{2}$, P. Nenoff ${ }^{1}$ \\ ${ }^{1}$ Klinik und Poliklinik für Hautkrankheiten der Universität Leipzig \\ 2 Institut für Pathologie der Universität Leipzig
}

\begin{abstract}
Zusammenfassung. Mastozytosen stellen eine sehr heterogene Krankheitsgruppe dar, die durch abnormales Wachstum und Anhäufung von Mastzellen in Haut, Knochenmark, Gastrointestinaltrakt, Leber, Milz und Lymphknoten gekennzeichnet ist. Symptome entstehen durch Freisetzung von Histamin, Prostaglandin D2, Heparin und anderen Mediatoren aus Mastzellen. Das Spektrum der klinischen Erscheinungsbilder reicht von isolierten Mastozytomen bis zu malignen Mastozytosen und der Mastzell-Leukämie. Anhand von zwei Fallbeispielen wird die variationsbreite Symptomatik verdeutlicht. Aufgrund der Vielfältigkeit der Erkrankung werden Lösungswege zur symptomorientierten Diagnostik und Therapiemöglichkeiten beschrieben. Differenzialdiagnosen und assoziierte hämatologische Erkrankungen werden nachfolgend diskutiert sowie die Wichtigkeit der Beratung und regelmäßigen Untersuchung der an Mastozytose erkrankten Patienten verdeutlicht.
\end{abstract}

Systemic Mastocytosis - Classification, Symptoms, Associated Diseases, Update Therapy Trends. Mastocytosis is a heterogeneous group of disorders characterised by an abnormal growth and accumulation of mast cells in skin, bone marrow, gastrointestinal tract, liver, spleen, and lymph nodes. Symptoms occur due to the release of histamine, prostaglandin D2, heparin and other mediators derived from mast cells. The clinical feature covers a broad spectrum from single mastocytoma to malignant mastocytosis and mast cell leukaemia. We report on two cases with adult benign systemic mastocytosis to show the variety of symptoms. Diagnostics oriented according to symptoms and options on therapy are presented as well as differential diagnosis and associated haematological disorders. The importance of advice and regular follow-ups of patients with systemic mastocytosis is emphasised.

\section{Einleitung}

Nachdem Paul Ehrlich 1877 über Bindegewebszellen mit Granula berichtete, die er Mastzellen nannte [1], beschrieb Nettleship 1889 erstmals die typischen Läsionen der Mastozytose,

Akt Dermatol 2001; 27: 53-57

(c) Georg Thieme Verlag Stuttgart · New York ISSN 0340-2541 die Urticaria pigmentosa, bei einem 2-jährigen Mädchen sowie das apfelsinenartig urtikarielle Anschwellen beim Reiben derselben (Darier-Zeichen) [2]. Die Mastozytose stellt ein sehr heterogenes Krankheitsbild unterschiedlicher Dignität dar, das durch eine pathologische Zunahme und Anhäufung von Mastzellen im Gewebe gekennzeichnet ist. Unter diesem Begriff wird ein breites Spektrum von Erkrankungen zusammengefasst, die von isolierten Hautinfiltraten (Mastozytomen) bis hin zur malignen Mastozytose und Mastzell-Leukämie reichen (Tab. 1) [3].

Im Folgenden berichten wir über zwei Patientinnen mit systemischer Mastozytose, die eine sehr unterschiedliche Symptomatik boten und sehr starken Leidensdruck aufgrund völlig verschiedener Aspekte dieser Erkrankung aufwiesen.

\section{Fallberichte}

Kasuistik 1

Anamnese

1989 waren der 37-jährigen Patientin erstmals rotbräunliche Knötchen an den Oberschenkelinnenseiten aufgefallen, die sich im Laufe eines Jahres auf den gesamten Körper ausbreiteten. Im Juni 1994 traten Schwindelanfälle während der Einnahme von Mahlzeiten auf, einmalig sei es sogar zu einer kurzen Bewusstlosigkeit gekommen.

\section{Hautbefund}

Bei der Erstvorstellung in der Universitäts-Hautklinik in Leipzig im Januar 2000 zeigten sich am gesamten Integument unter Aussparung des Gesichts, der Palmae und Plantae in unterschiedlicher Dichte disseminierte, unscharf begrenzte plane, teils wenig erhabene rosa bis rötlichbraune, linsen- bis erbsengroße Maculopapeln mit gelegentlichem Pruritus. Das DarierZeichen war positiv.

\section{Histologie}

Probebiopsie linker Oberschenkel: Es imponierten diskret ausgeprägte gemischtzellige entzündliche Infiltrate aus mononukleären Zellen und einzelnen eosinophilen Granulozyten, die umschlossenen Gefäße wiesen eine geringgradige Dilatation auf. In den Spezialfärbungen Giemsa und Toluidin- 
Tab.1 Klassifikation der Mastozytosen

Benigne Mastozytosen

Kutan:

Isoliert: Mastozytom

Generalisiert: Urticaria pigmentosa

Diffuse Mastozytose

Systemisch:

Haut (nicht obligatorisch)

Knochenmark (Osteosklerose, Osteomalazie)

Leber

Milz (Hypersplenismus mit thrombo-zytopenischer Purpura)

Lymphknoten

Gastrointestinaltrakt (Anorexie, Bauch- und Kopfschmerzen,

Schwindel, Erbrechen, Diarrhöen, Magenulzera)

$\rightarrow$ Kein Nachweis von Mastzellen im Blut!

Maligne Mastozytosen

Mastzell-Leukämie (Infiltration des Knochenmarks und anderer Organe, fakultativ: Hautbeteiligung)

$\rightarrow$ Nachweis unreifer zirkulierender Mastzellen im Blut!

blau ließen sich innerhalb der Entzündungszellinfiltrate Mastzellen identifizieren.

Jamshidi-Punktion des Knochenmarks: Nachweis von Proliferationsnestern von Mastzellen.

Probeexzisionen der Magen- bzw. Duodenalschleimhaut: geringe chronische Gastritis der Antrumschleimhaut mit basaler Fibrose und leichter Mastzellvermehrung sowie Mastzellhyperplasie der Corpusschleimhaut des Magens. Im Duodenum ließ sich eine diskrete Duodenitis mit leichter Mastzellhyperplasie nachweisen.

\section{Weitere Befunde}

Routine-Laborparameter, Blutdruckwerte, Röntgen-Thorax, Oberbauchsonographie und Knochenmarkzytologie unauffällig.

\section{Diagnose}

Benigne adulte systemische Mastozytose mit Haut-, Gastrointestinal- und fraglicher Knochenmarkbeteiligung ohne Zeichen der malignen Entartung.

\section{Therapie und Verlauf}

Die Patientin wurde darüber aufgeklärt, dass es bei Gabe von mastzelldegranulierenden Medikamenten (Tab. 2), durch Freisetzung von Histamin und anderen Mediatoren zu systemischen Reaktionen bis hin zum anaphylaktischen Schock kommen kann. Dass auch stärkere physikalische Reize (Hitze, Kälte, Sonnenlicht, Reibung), Bakterientoxine, Schlangen- und Hymenopterengifte sowie biologische Polypeptide, die beispielsweise von Quallen, Krebsen, Langusten und Hummern freigesetzt werden, Symptome hervorrufen können, wurde ebenfalls mitgeteilt. Bei der Patientin wurde eine orale PUVATherapie durchgeführt, die insofern erfolgreich war, als das
Tab.2 Histaminliberatoren, bzw. Mastzelldegranulatoren

Zentral wirkende Stoffe/Muskelrelaxantien/Narkotika
Atropin
Codein
Gallamin
Morphin
Papaverin
Scopolamin
Tubocurarin
Sympathominmetika
Amphetamin
Tyramin
Alle nichtsteroidalen Antirheumatika inkl. Aspirin
Antihypertonika
Hydralazin
Tolazolin
Chemotherapeutika
Pentamidin
Aminoglykoside
Sonstige
Gelatine
Röntgenkontrastmittel
Chinin
Quinine
Thiamin
Dextran
Alkohol

Darier-Zeichen unterdrückt wurde und die Hauterscheinungen regredient waren. Dieser Therapieerfolg hält bereits seit mehreren Monaten an.

\section{Kasuistik 2}

\section{Anamnese}

Bei der 42-jährigen Patientin ist bereits seit 1988 eine Urticaria pigmentosa bekannt, die bislang keiner spezifischen Therapie bedurfte. Seit einiger Zeit klagte die Frau über ein allgemeines Gefühl der Abgeschlagenheit, Kopfschmerzen, Übelkeit, Schwindelgefühl und Durchfälle.

\section{Hautbefund}

Bei stationärer Aufnahme sah man bräunlich bis rötliche 1 $2 \mathrm{~mm}$ große Papeln mit positivem Darier-Zeichen am gesamten Integument unter Betonung des Rumpfes und Aussparung der Hand- und Fußflächen sowie des Gesichtes (Abb.1).

\section{Histologie/Immunhistologie}

In der Probebiopsie einer Läsion vom Thorax fanden sich an umschriebener Stelle im Stratum papillare und oberem Korium bandförmige Vermehrungen von Mastzellen. Die Becken- 


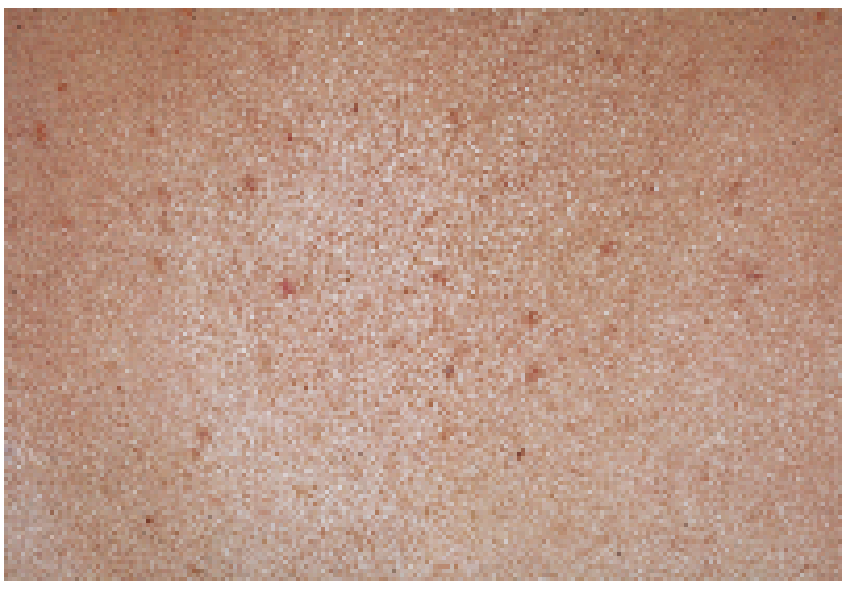

Abb.1 Hautbeteiligung bei systemischer adulter benigner Mastozytose.

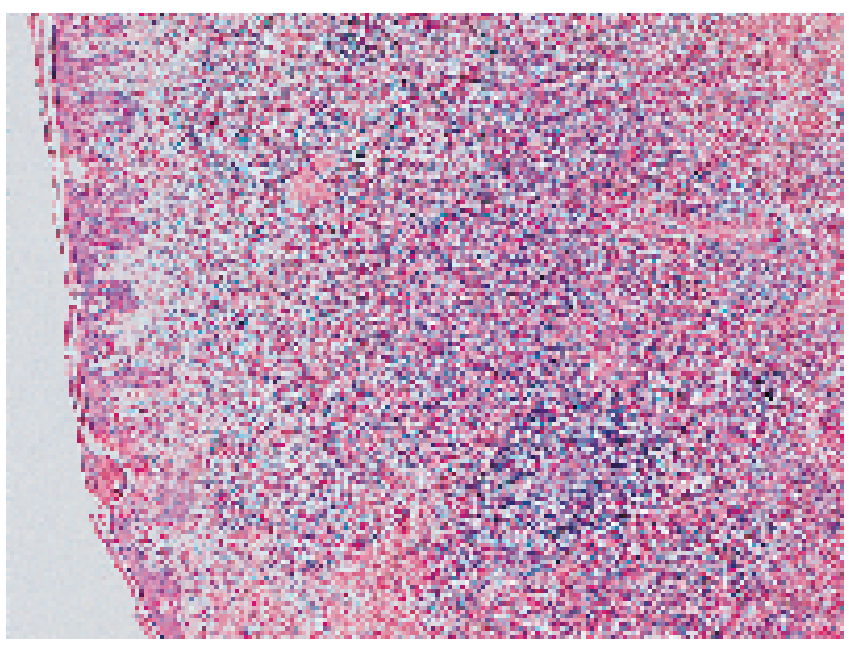

Abb.2 Ausgeprägte Infiltration des Knochenmarks durch Mastzellen (Giemsa, 250x), Ausschnitt: Immunreaktion mit anti-MastzellenAntikörper (AA1, APAAP-Reaktion, 250x).

kammbiopsie zeigte nodulär akzentuierte gemischt lymphozytär mastozytäre Infiltrate, die insgesamt $20 \%$ der Fläche und $25 \%$ der kernhaltigen Zellen ausmachten. Die Mastzellinfiltrate lagen zum Teil granuliert, teils degranuliert vor. Die Zahl der Eosinophilen war zusätzlich stark vermehrt (Abb. 2). Die Mastzellen reagierten stark mit dem anti-Mastzellantikörper (AA1, siehe Abb. 2, Ausschnitt).

\section{Weitere Befunde}

In der Ösophago-Duodenoskopie und Koloskopie fand sich kein Hinweis für eine systemische Mastozytose. In der Ganzkörper-Skelett-Szintigraphie konnte eine geringgradige Osteoporose detektiert werden. Oberbauchsonographie, RöntgenThorax und Routinelabor erwiesen sich als unauffällig. Andere Ursachen für Schwindel, Übelkeit und Diarrhöen wurden ausgeschlossen.

\section{Diagnose}

Benigne adulte systemische Mastozytose mit Haut- und Knochenmarkbeteiligung mit assoziierter Osteoporose und ohne Hinweise für eine maligne Entartung.

\section{Therapie und Verlauf}

Über die Möglichkeit der Mastzelldegranulation durch Medikamente und mechanische Reize wurde aufgeklärt. Am störendsten wurden das Schwächegefühl und der massive Leistungsabfall empfunden, die Hautsymptome waren für die Patientin unbedeutend. Es wurde nochmals auf die Gutartigkeit der Erkrankung hingewiesen, bezüglich der Übelkeit wurde sie mit Ranitidin behandelt und erhielt Etidronsäure zur Verhinderung des Knochensubstanzverlustes.

Eine Therapie mit Interferon alpha-2a wurde in Erwägung gezogen, aber aufgrund der geringen Ausprägung des Krankheitsbildes nicht eingeleitet.

\section{Besprechung}

Anhand der beiden Fallbeispiele wird deutlich, wie breit gefächert die Symptome bei systemischer Mastozytose in Abhängigkeit von der bestehenden Organbeteiligung sein können und wie schwierig sich die Diagnosestellung gestalten kann.

Die typischen Symptome bei systemischer Mastozytose können interindividuell variieren und sich im gleichen Patienten in der Intensität im Laufe der Zeit ändern. Viele Erkrankte beschreiben das Auftreten der so genannten Flush-Symptomatik, teils als zufälliges Ereignis, teils als Hauptquelle des Unwohlseins, ebenso wie das Vorhandensein von Kopfschmerzen unterschiedlicher Intensität. Die meisten klagen über den massiven Pruritus, manche über Quaddelbildung; in seltenen Fällen kann sogar eine Blasenbildung auftreten [4]. Die häufigsten chronischen Beschwerden finden sich im Magen-Darm-Trakt in Form gastrointestinaler Ulzera, Gastritis/Duodenitis, Übelkeit, Erbrechen, Diarrhöen, Malabsorption mit konsekutiver Hypokalzämie und Hypomagnesiämie bis hin zur nachfolgenden Tetanie [5].

Das Auftreten dieser Symptome ergibt sich aus der Mastzellinfiltration des jeweiligen Organparenchyms. In der Literatur finden sich diesbezüglich sehr unterschiedliche Angaben. Systemische Mastozytosen, das heißt der Befall mindestens eines extrakutanen Organs, sind insgesamt verhältnismäßig selten. In ca. 5-10\% der Patienten mit isolierten Mastozytomen und Urticaria pigmentosa infantum oder adultorum sowie bei etwa $40 \%$ der Patienten mit diffuser, beziehungsweise erythrodermischer Mastozytose liegt eine Systembeteiligung vor [6]. Die Haut (>90\%) und das Knochenmark (bis zu 90\%) sind am häufigsten betroffen, Hepato- und Splenomegalie treten bei 12 $70 \%$ der Patienten, Lymphknotenvergrößerungen bei $10-37 \%$, ein gastrointestinaler Befall in ca. 5-25\% aller Fälle von systemischer Mastozytose auf. Eine Nierenbeteiligung ist extrem selten, während 1-5\% Mastzell-Leukämien vorliegen [7 -9].

Häufig suchen die betroffenen Patienten zunächst den Dermatologen aufgrund der Hautsymptomatik auf. Eine Hautbiopsie ist neben dem typischen oben beschriebenen klinischen Bild zur Diagnosesicherung einer Urticaria pigmentosa unerläss- 
lich, um die histologisch charakteristischen Mastzellproliferate in der Giemsa- oder Toluidinblau-Färbung darstellen zu können. Das Darier-Zeichen (urtikarielle bis blasige Effloreszenzen nach Reiben der bestehenden Herde) ist ein nützlicher diagnostischer Hinweis und hilft, differenzialdiagnostisch zur Unterscheidung von anderen tumorartigen Hauteffloreszenzen, Langerhanszell-assoziierten Histiozytosen, Lymphomen, Leiomyomen, Xanthomen, Arzneimittelexanthemen und Insektenstichreaktionen.

Eine systemische Mastozytose sollte durch entsprechende Diagnostik ausgeschlossen oder bestätigt werden. Da das Krankheitsbild sich als außerordentlich vielfältig erwiesen hat, sollte man sich an den bestehenden Symptomen orientieren und neben der grundlegenden Diagnostik, die nach Langer [8] eine Probeexzision, Röntgen-Thorax, Skelettröntgen, beziehungsweise Skelettszintigraphie, eine Abdomensonographie und Laboruntersuchungen (Differenzialblutbild, Thrombozyten, Gerinnungsparameter, Blutsenkung, Elektrophorese, Gesamt-IgE und Blutchemie) beinhaltet, nur bei begründetem Verdacht der Organbeteiligung die Basisdiagnostik erweitern und beispielsweise eine Beckenkammbiopsie, endoskopische oder radiologische Magen- und Darmuntersuchungen, neurologische Diagnostik oder Lungenfunktion anschließen.

Bei gezielter differenzialdiagnostischer Fragestellung ist die Bestimmung des Histamins und seiner Metaboliten, die Ausscheidung der 5-Hydroxyindolessigsäure und die Katecholaminausscheidung im 24-h-Sammelurin möglich [8].

Eine wichtige Differenzialdiagnose der systemischen Mastozytose ist das Karzinoidsyndrom, bei dem sich eine ähnliche Symptomatik zeigt: Beide Erkrankungen gehen mit spontanem episodischen Erröten (Flush), unspezifischen gastrointestinalen Beschwerden, Tachykardie, Hypotension und Hepatomegalie einher. Ferner sollte auch an das Vorliegen eines Phäochromozytoms als Ursache der Flushsymptomatik und Nahrungsmittelunverträglichkeiten in Betracht gezogen werden [6].

Die Behandlung der Mastozytose erfordert die Kenntnis der Pathomechanismen, um hierauf basierend eine adäquate Therapie entsprechend des vorliegenden Krankheitsmusters wählen zu können. Eine kausale Intervention ist bisher noch nicht möglich. Die Therapie der meisten Mastozytosen erfolgt konservativ und symptomatisch und richtet sich nach den subjektiven Beschwerden des Patienten, die durch die Mediatorfreisetzung von Mastzellen hervorgerufen werden.

Medikamentöse Therapie der Wahl ist die Gabe von H1- und H2-Antihistaminika in Kombination oder einzeln [10,11]. Zeitweise wurde die Kombinationstherapie favorisiert [12], Therapieversager sind jedoch beschrieben worden [13]. Insbesondere die kutanen Symptome werden durch Antihistaminika positiv beeinflusst.

Die gastrointestinalen Beschwerden sind am ehesten mit Dinatriumcromoglykat bei prophylaktischer Anwendung zu beherrschen. Es blockiert die Histaminfreisetzung aus Mastzellen, wirkt sich aber weniger effektiv auf die Minderung der Hautsymptome aus [14]. Als weiterer Mastzellstabilisator und gleichzeitig Antihistaminikum sei Ketotifen genannt, das durch gute Resorption beide Symptomenkomplexe, MagenDarm-Trakt und Haut, gut beeinflusst [15].

Vella et al. [16] und Christophers et al. [17, 18] berichten über die gute Wirksamkeit von oraler Photochemotherapie (PUVA) mit 8-Methoxypsoralen in Bezug auf das Ausmaß der Hauterscheinungen, den Juckreiz sowie die Provozierbarkeit von Quaddeln (Darier-Zeichen) mit unterschiedlich lange anhaltendem Therapieerfolg (wenige Wochen bis zu zehn Jahre) ohne direkte Dosis-Wirkungs-Beziehung. Bade-PUVA hat sich in vier Fällen mit Urticaria pigmentosa als unwirksam erwiesen [18].

Hautbefunde bei systemischer Mastozytose können durch topische Steroide beziehungsweise Triamcinoloninjektionen zur Abheilung gebracht werden [19].

Acetylsalicylsäure und andere nichtsteroidale Antirheumatika (z. B. Ibuprofen und Diclofenac), die als Prostaglandin-Syntheseinhibitoren dienen, werden bei rezidivierend auftretender Flushsymptomatik angewandt. Die Verwendung dieser Substanzen sollte jedoch genau abgewogen werden, da unspezifische Mastzelldegranulationen mit Histaminsymptomatik vorkommen können und auch die häufig vorhandene Hyperazidität des Magens verstärkt werden kann [10].

Anticholinergika (Atropin) kommen bei abdominalen Schmerzen und Krämpfen zum Einsatz [10, 20].

Ein dermatologischer Notfall sind die potenziell lebensbedrohlichen Folgen einer verstärkten Mediatorenfreisetzung aus aktivierten Mastzellen. Sie kann zu Hypotonie und akuten kollaps- oder schockartigen Ereignissen führen, die mit Antihistaminika nicht beherrschbar sind. In solchen Fällen ist der Einsatz von Sympathomimetika indiziert. Epinephrin ist geeignet, eine gravierende Hypotension $\mathrm{zu}$ beheben, bei schweren Schockzuständen ist Adrenalin das Mittel der Wahl. Sollte sich nach Bolusinjektion nur eine kurzfristige Blutdrucksteigerung erreichen lassen, ist eine Adrenalindauerinfusion mittels Perfusor angezeigt [21].

In der Literatur sind Fallberichte über systemische Mastozytosen, bei denen Interferon-alpha injiziert wurde, beschrieben. Interferone (IFN- $\alpha$ und IFN- $\gamma$ ) inhibieren das Wachstum von Mastzellen in vitro, woraus sich der Therapieansatz bei myeloproliferativen Erkrankungen ableitet [22]. Neben den aggressiven Formen wurden inzwischen auch Patienten mit Urticaria pigmentosa und benigner adulter systemischer Mastozytose mit gutem Erfolg behandelt [23-26]. Die Hautläsionen sprachen auf diese Therapie teilweise nicht an [23]. Die im zweiten Fallbericht beschriebene Patientin wurde nicht mit Interferon$\alpha$ therapiert, da die symptomatische Therapie der Patientin ausreichend erscheint, um Durchfälle und kardiovaskuläre Ereignisse zu unterdrücken und zudem keinerlei Blutbildveränderungen vorliegen, die eine IFN- $\alpha$-Therapie rechtfertigen würden.

Aggressivere Therapieformen wie Zytostatika (Chlorambucil und Stickstofflost, [3]) und Splenektomie zur Verminderung der Thrombozytopenie und besserer Verträglichkeit nachfolgender Chemotherapien [9, 27, 28] sollten den malignen Mastozytosen, der Mastzell-Leukämie und assoziierten hämatologischen Erkrankungen vorbehalten bleiben. 
Die prognostische Einschätzung der systemischen Mastozytose ist schwierig, aber nicht unerheblich. Häufig ist der Zustand bei adulter benigner Mastozytose über lange Zeit stabil. Dennoch gibt es einige Faktoren, die als prognostisch ungünstig einzustufen sind. Schwerwiegende systemische Beteiligung scheint mit einem Erkrankungsbeginn im höheren Lebensalter, einem ausgedehnten Hautbefall und besonders mit dem Vorliegen diffuser oder erythrodermischer Mastozytosen zu korrelieren [29, 30]. Sagher et al. [3] haben festgestellt, dass das Risiko der Entwicklung einer Systembeteiligung bei Urticaria pigmentosa 8- bis 9-mal höher ist, wenn sich die Erkrankung erstmalig im Erwachsenenalter manifestiert.

Die systemische Mastozytose kann mit Leukämien und Lymphomen einher gehen; selten tritt eine Mastzell-Leukämie auf, jedoch wird über Fälle systemischer Mastozytose und gleichzeitiger myeloischer oder monozytärer Leukämie, Osteomyelofibrose, M. Hodgkin, Polycythaemia vera oder Non-Hodgkin-Lymphomen berichtet. Die assoziierten Malignome treten nicht regelmäßig auf, so dass es ausschließlich Fallberichte gibt und eine statistische Absicherung fehlt. Lediglich die Koexistenz der Leukämie in 4-5\% der Patienten mit systemischer Mastozytose wurde festgestellt [31].

Dieser Zusammenhang unterstreicht die Notwendigkeit von Kontrolluntersuchungen, auch wenn die Assoziation zu malignen hämatologischen Erkrankungen selten ist.

Anhand der hier vorgestellten Fälle von adulter benigner Mastozytose wird deutlich, wie breit gefächert das klinische Spektrum dieser ungewöhnlichen und heterogenen Erkrankung sein kann und wie wichtig es ist, andere Erkrankungen abzugrenzen, beziehungsweise assoziierte Erkrankungen auszuschließen. Weiterführende Studien und Forschungen bezüglich der Mastzellherkunft, -funktion und -regulation werden zur Klärung der Pathogenese der Mastozytose sicherlich beitragen und möglicherweise neue Therapieoptionen eröffnen.

\section{Literatur}

${ }^{1}$ Ehrlich P. Beiträge zur Kenntnis der Anilinfärbungen und ihrer Verwendung in der mikroskopischen Technik. Arch Mikr Anat 1877; 13: $263-264$

${ }^{2}$ Metcalfe DD. Classification and diagnosis of mastocytosis: current status. J Invest Dermatol 1991; 96: 3, 2S-4S

${ }^{3}$ Sagher F, Even-Paz Z. Mastocytosis and the mast cell. In: Year Book Medical Publishers, Inc., Chicago: 1967: 4-242

${ }^{4}$ Haustein UF. Bullöse Mastozytose bei einem Kind. Hautarzt 1997; 48: $127-129$

${ }^{5}$ Horan RF, Austen KF. Systemic mastocytosis: retrospective review of a decade's clinical experience and the Brigham and Women's hospital. J Invest Dermatol 1991; 96: 5S - 14S

${ }^{6}$ Amon U. Mastozytosen. In: Braun-Falko O, Plewig G, Wolff $\mathrm{HH}$ (Hrsg) Dermatologie und Venerologie. Berlin, Heidelberg, New York: Springer, 1997: 1431 - 1438

${ }^{7}$ Valent P. Biology, classification and treatment of human mastocytosis. Wien Klin Wochenschr 1996; 108: 385 - 397

${ }^{8}$ Langer K, Wolff K. Das klinische Spektrum der Mastozytosen. Hautarzt 1990; 41: 188-195

${ }^{9}$ Travis WD, Li CY, Hogaland HC, Travis LB, Banks PM. Mast cell leukemia: Report of a case and review of the literature. Mayo Clin Proc 1986; 61: 957-966

${ }^{10}$ Metcalfe DD. The treatment of mastocytosis: an overview. J Invest Dermatol 1991; 96: 55S-59S
${ }^{11}$ Gasior CB, Falk ES. Systemic mastocytosis treated with histamine H1 and H2 receptor antagonists. Dermatology 1992; 184: 149

${ }^{12}$ Gerrard JW. Urticaria pigmentosa: treatment with cimetidine and chlorpheniramine. J Pediatr 1979; 94: 843-844

${ }^{13}$ Horan RF, Sheffer AL, Austen KF. Cromolyn sodium in the management of systemic mastocytosis. J Allergy Clin Immunol 1990; 85: $852-855$

${ }^{14}$ Frieri M, Alling DW, Metcalfe DD. Comparison of the therapeutic efficacy of cromoyn sodium with that of combined chlorpheniramine and cimetidine in systemic mastocytosis: results of a double-blind clinical trial. Am J Med 1985; 78: 9-14

${ }^{15}$ Czarnetzki BM. A double-blind cross-over study of the effect of ketotifen in urticaria pigmentosa. Dermatologica 1983; 166: 44-47

${ }^{16}$ Vella BD, Eady RA, James MP, Gatti S, Bleehen SS. Photochemotherapy (PUVA) in the treatment of urticaria pigmentosa. Br J Dermatol 1983; 109: 67 - 75

${ }^{17}$ Christophers E, Hönigsmann H, Wolff K, Langner A. PUVA treatment of urticaria pigmentosa. Br J Dermatol 1978; 98: 701 - 702

${ }^{18}$ Godt O, Proksch E, Streit V, Christophers E. Short- and long-term effectiveness of oral and bath PUVA therapy in urticaria pigmentosa and systemic mastocytosis. Dermatology 1997; 195: 35-39

${ }^{19}$ Barton J, Lavker RM, Schechter NM, Lazarus GS. Treatment of urticaria pigmentosa with corticosteroids. Arch Dermatol 1985; 121: $1516-1523$

20 Orfanos CE, Garbe C. Mastzellkrankheiten. In: Therapie der Hautkrankheiten. Heidelberg, New York: Springer Berlin, 1995: 549

${ }^{21}$ Reinhold U, Dill-Müller D, Koch P. Mastozytosesyndrom. In: Notfälle in der Dermatologie. Stuttgart: Thieme, 1999: 146-148

22 Bissonnette EY, Chin B, Befus AD. Interferons differentially regulate histamine and TNF-alpha in rat intestinal mucosa cells. Immunology 1995; 86: 12 - 17

${ }^{23}$ Kissling S, Kernland K, Gerbig AW, Hunziker T. Strategies in childhood and adult mastocytosis. Dermatology 1999; 198: 426-430

${ }^{24}$ Krahl J, Baldauf P, Stoermer D. Systemische konnatale Mastozytose mit universeller Hautbeteiligung. Hautarzt 1999; 5: 893-896

${ }^{25}$ Hubner C, Wedding U, Strater J, Limberg B, Stremmel W. Clinical stable systemic mastocytosis with interferon alpha- $2 b$ therapy. J Intern Med 1997; 241: 529-533

${ }^{26}$ Kolde G, Sunderkotter C, Luger TA. Treatment of urticaria pigmentosa using interferon alpha. Br J Dermatol 1995; 133: 91 - 94

${ }^{27}$ Friedman B, Darling G, Norton J, Metcalfe DD. Splenectomie in the management of systemic mast cell disease. Surgery 1990; 107: $94-100$

${ }^{28}$ Czarnetzki BM, Kolde G, Schoemann A, Urbanitz S, Urbanitz D. Bone marrow findings in adult patients with urticaria pigmentosa. J Am Acad Dermatol 1988; 18: 45 - 47

${ }^{29}$ Parker F, Odland GF. The mastocytosis syndrome. In: Fitzpatrick TB, Arndt KA, Clark WH Jr et al (Hrsg). Dermatology in general medicine. Mc Graw Hill Book Co, 1979: 772 - 783

${ }^{30}$ Klaus SN, Winkelmann RK. Course of urticaria pigmentosa in children. Arch Dermatol 1962; 86: 116-119

31 Di Bacco RS, DeLeo VA. Mastocytosis and the mast cell. J Am Acad Dermatol 1982; 7: 709-722

\section{Dr. med. Christina Rogalski}

Universitätsklinikum Leipzig AöR

Klinik und Poliklinik

für Hautkrankheiten

Liebigstraße 21

04103 Leipzig

E-mail: nenp@server 3.medizin.uni-leipzig.de 